\title{
PREDICTION OF SUGARCANE QUALITY FROM JUICE SAMPLES USING PORTABLE SPECTROSCOPY
}

\author{
N.M. Nawi ${ }^{1,2 *}$, G. Chen ${ }^{2}$ and T. Jensen ${ }^{2}$ \\ ${ }^{1}$ Department of Biological and Agricultural Engineering, Faculty of Engineering, \\ Universiti Putra Malaysia, 43400 UPM, Serdang, Selangor, Malaysia \\ *Email: nazmimat@upm.edu.my \\ Phone: +60389464331; Fax: +60389464325 \\ ${ }^{2}$ Faculty of Engineering and Surveying, University of Southern Queensland, \\ Toowoomba, QLD 4350, Australia
}

\begin{abstract}
Rapid determination of sugarcane quality using low-cost and portable equipment is more practical for field use. Thus, this study explored the potential application of a portable visible and shortwave near infrared spectroradiometer (VNIRS) to predict pol and brix from sugarcane juice samples. A total of 100 sugarcane juice samples for each clear and raw juice samples were assessed. The spectral data were collected by scanning the juice samples in a cuvette with $10 \mathrm{~mm}$ path length using transmittance mode. Partial least squares (PLS) and principal component analysis (PCA) were applied to interpret the spectra and develop both calibration and prediction models. The prediction performances for the clear juice samples were good with coefficient of determination $\left(\mathrm{R}^{2}\right)$ values of pol and brix were 0.85 and 0.84 , respectively. For the raw juice samples, the prediction performances were acceptable with $\mathrm{R}^{2}$ values for pol and brix were 0.73 and 0.74 , respectively. Based on these results, it was concluded that the VNIRS combined with PLS models could be applied to predict sugarcane quality from both clear and raw sugarcane juices.
\end{abstract}

Keywords: VNIRS; spectroscopy; sugarcane; quality; juice samples.

\section{INTRODUCTION}

Sugarcane (Saccahrum spp.) is an important crop in Australia with the production value ranging from AUD\$1.5 to 2.5 billion per year (Canegrowers, 2011). In the Australian sugarcane industry, growers are paid based on both yield and quality of their product. Sugarcane quality is determined based on its sugar content, known as commercial cane sugar (CCS). CCS is derived from brix (soluble solids content), pol (sucrose content) and fibre content. The brix and pol are usually measured in a laboratory using conventional measurement methods including refractometer, polarimeter and chromatographer. Lately, the applications of laboratory spectroscopic methods as rapid and simple measurement systems in measuring sugarcane quality parameters have been reported [1-3]. Recently, there is a growing interest within the industry to measure sugarcane quality in the field. Ability to measure sugarcane quality in the field will be very useful to the industry for assessing the crops growth and development, harvesting management and adoption of the precision agriculture technique. Unfortunately, the conventional measurement methods and laboratory spectroscopic methods have great limitations for field uses because they are often time-consuming, operator dependent, 
and require hazardous reagents [1]. Moreover, these laboratory technologies are not suitable to be used in the field considering the potential damage due to harsh and dusty environment in the field. This laboratory equipment is also expensive, thus should be handled with a great care. Therefore, a portable, robust and low-cost spectrometer is more preferable for field use. A portable visible and shortwave near infrared spectroradiometer (VNIRS) with a wavelength range from 350 to $1100 \mathrm{~nm}$ appears promising for predicting fruit quality since the instrument is low-cost and portable enough for in-field measurements [4]. The applications of this equipment to measure sugarcane quality from stalk samples have been reported by Nawi, Jensen [5], Mat Nawi, Chen [6] and Mat Nawi, Chen [7]. However, no study has reported the use of this equipment to measure sugarcane quality from juice samples. The application of this equipment seems to be feasible because it is small, portable and low-cost compare to other spectroscopy sensors. Therefore, the objectives of this study were (1) to investigate the feasibility of using VNIRS to predict brix and pol in sugarcane juice samples; (2) to compare the prediction performance between clear and raw juice samples.

\section{MATERIALS AND METHODS}

\section{Sugarcane Juice Samples}

A total of 100 juice samples were collected for each clear and raw sugarcane juices. Each juice sample was extracted from a sample set consisted of a group of six whole stalk samples. Each sample set was collected from different field trials in Bundaberg, Australia throughout the harvest season in 2012. The selection of the sample sets from different harvest locations helped to establish sufficiently robust calibration models that are applicable to all varieties. Each sample set was passed through the Spectracane system at the Bureau of Sugar Experimental Station (BSES) research station in Bundaberg, Queensland, Australia. Each sample set was first fibrated using a sugarcane disintegrator (Dedini, Model D-2500-II) with a $10 \mathrm{Hp}, 400 \mathrm{~V}$ motor and operating at $3340 \mathrm{rpm}$. The first expressed juice was collected by hydraulically pressed the fibrated sugarcane samples at $25 \mathrm{MPa}$ for 1 minute. Then, the collected juices of each sample set were filtered and divided into two sets, one to determine brix and another one to determine pol. The brix measurement was done on raw juices using a Bellingham and Stanley RFM 310 refractometer. For pol measurement, the raw juice samples were first clarified using lead acetate to produce clear juices (Figure 1). Pol measurement was done on clear juice samples using a Polartronic Universal automatic polarimeter (Schmidt + Haensch, Berlin, Germany).

\section{Instrument and Spectral Collection}

The transmission spectra of the samples were measured using a handheld VNIRS (FieldSpecHandHeld and FieldSpec Pro FR, 325 to $1075 \mathrm{~nm}$, Analytical Spectral Devices (ASD), Inc., Boulder, USA). The spectral measurements were conducted inside a black measurement box $(900 \times 600 \times 450 \mathrm{~mm})$. The box was built to enclose the sensor and the samples from the ambient light (Figure 2). The juice samples were placed into a plastic cuvette with $10 \mathrm{~mm}$ of optical path length. The field of view (FOV) of the spectroradiometer was $10^{\circ}$, giving a working distance of $45 \mathrm{~mm}$ from the cuvette 
with the diameter of the scanning area of $10 \mathrm{~mm}$. A cuvette holder was built to firmly hold the cuvette inside the box throughout the experiments. One side of the cuvette was scanned using the sensor while on another side was illuminated with halogen lamp (Lowell Pro-Lam 14.5 V tungsten bulb, Ushio Lighting, Inc., Japan). The lamp was placed about $300 \mathrm{~mm}$ away from the measurement samples.

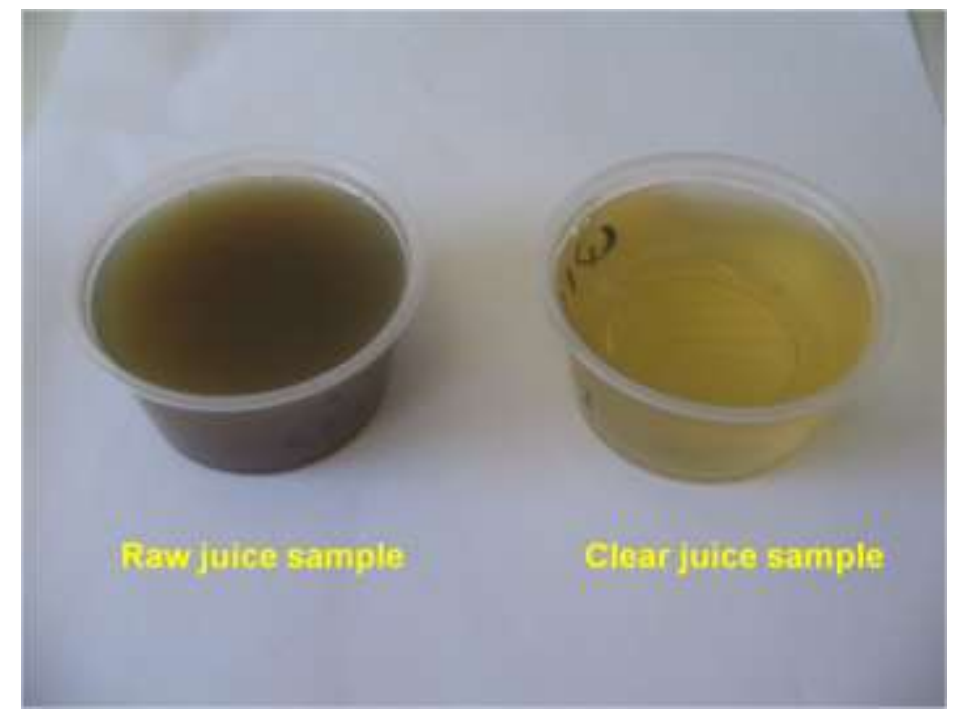

Figure 1. A comparison between raw and clear juice samples.

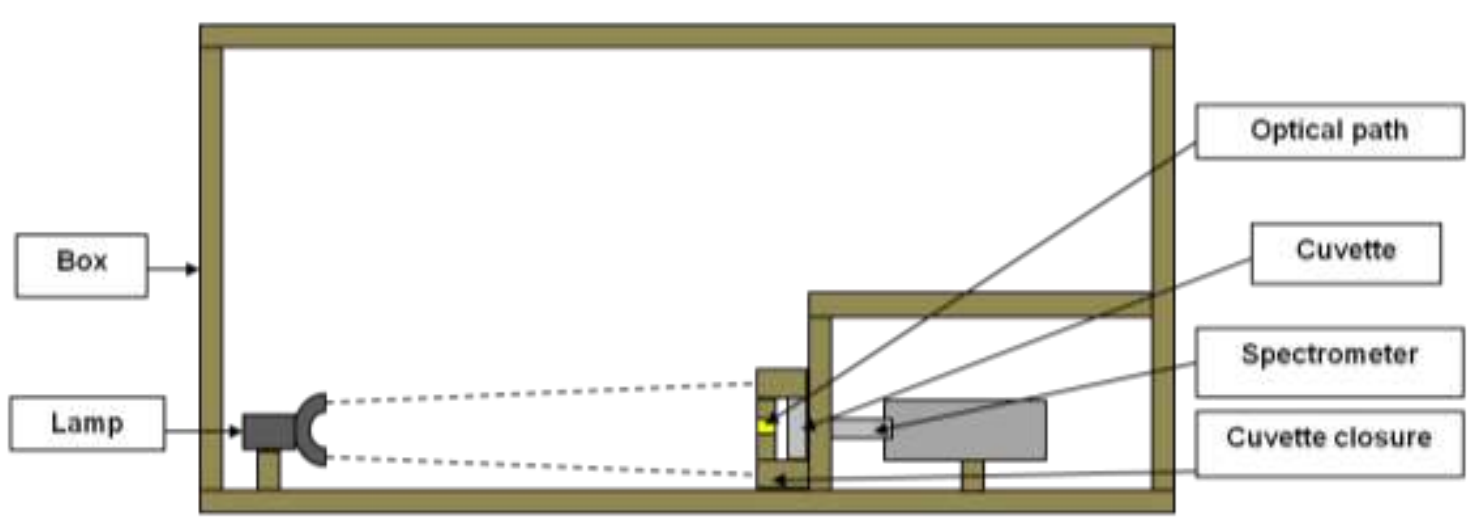

Figure 2. Simplified diagram of the transmittance measurement inside the measurement box

The reference spectrum was acquired using the cuvette filled with distilled water and the dark spectrum was acquired when the lamps were off. Transmission spectra from 375 to $1075 \mathrm{~nm}$ were measured at $1.5 \mathrm{~nm}$ intervals with an average reading of 20 scans per spectrum. All spectral data were stored in a computer and processed using the RS3 software for Windows (Analytical Spectral Devices, Inc., Boulder, Colo.), designed with a graphical user interface. The transmission spectra were transformed into ASCII format by using the ASD ViewSpecPro software (Analytical Spectral Devices, Boulder, USA). Then, three spectra for each sample were averaged into one spectrum and transformed by $\log (1 / \mathrm{T})$ into absorbance spectrum. 


\section{Multivariate Analysis}

Before the calibration, the spectral data was pre-processed for optimal performance. Pre-processing of spectral data is a key part of spectral analysis to improve the accuracy. In this study, the effect of several pre-processing techniques on calibration model performance was investigated including smoothing technique of moving average, multiplicative scatter correction (MSC), first and second derivatives, standard normal variate (SNV) transformation and mean normalisation. After some trials and computations, MSC was found to be the best pre-processing technique for this study. In fact, MSC is the most popular normalisation technique offered by most chemometrics software packages. MSC was used to correct the light scattering variations in the spectral data [8]. The pre-processing processes were implemented using The Unscrambler, V 9.6 software (Camo Process AS, Oslo, Norway).

Prior to the development of calibration model, principal component analysis (PCA) was applied in analyzing the spectra data to extract useful information, decrease the noise and reduce the number of principle components (PCs). PCA is a well-known chemometrics method used to search for directions of maximum variability in sample grouping and uses them as new axes called principle components (PCs) that can be used as new variables, instead of the original data, in the following calculations (Blanco \& Villarroya, 2002). PCA was also used to detect spectral outliers that might affect model performance in each data set [9]. Two outliers were found in this study and they were removed prior to spectral analysis using partial least square (PLS) regression.

Partial least squares (PLS) analysis is commonly applied in the near infrared spectroscopy analysis. PLS analysis could be used to establish a regression model to perform the prediction of sugar content from sugarcane juices. PLS simultaneously considers the variable of matrix Y (brix and pol) and the variable of matrix X (spectral data). In this paper, PLS was applied as a regression method as well as a way to extract the latent variables (LVs). The LVs were considered as new eigenvectors of the original spectra to reduce the dimensionality and compress the original spectra data [10]. The maximum numbers of LVs used in this study was set to ten. Due to a limited number of samples used, full cross validation (leave-one-out) was used to evaluate the prediction quality and prevent over fitting of the calibration model [11]. External validation method was also used in this study to check the performance of the PLS models. The external validation procedure determines the predictive ability of an equation, based on a sample set which has not been used in the calibration development. Before the calibration, samples were divided into two sets. One set (75\% of samples) was used to develop a prediction equation (calibration set) and another set (25\% of samples) was used to validate the predictive equation (validation set). Samples for validation were selected by taking one of every four samples from the entire sample set, taking care to ensure that each set included samples that covered the entire range of sugarcane quality values. In this paper, both PCA for PLS modeling were run using the Unscrambler V 9.6. The performance of the final PLS models was evaluated by the coefficient of determination for calibration $\left(\mathrm{R}^{2}\right)$, root mean squares of calibration (RMSEC), and the coefficient of determination for prediction $\left(\mathrm{R}^{2}\right)$ and root mean squares of prediction (RMSEP). A proper model should have a low RMSEC, RMSEP and a high $\mathrm{R}^{2}$ for both calibration and prediction models. 


\section{RESULTS AND DISCUSSION}

\section{Overview of the Spectra and Statistical Values of Brix and Pol}

The typical absorbance spectra for each raw and clarified juice are shown in Figure 3. Both curves show a downward trend as the wavelength increased. A higher absorption level by raw juices was due to the presence of impurities, colour pigments and fine fibers [12]. At $680 \mathrm{~nm}$, spectra curve for raw juice displayed a weak peak while this peak was not observed for clear juice curve. The peak at $680 \mathrm{~nm}$ could be related to chlorophyll pigments [13]. For the clarified juice sample, the chlorophyll content was eliminated through clarification process. For the raw juice sample, it can be seen that there was some noises in the range of 400-550 $\mathrm{nm}$. Hence, to afford better comparison with better prediction performance, only the wavelength region from 600 to $1000 \mathrm{~nm}$ was used for the analysis in this study.

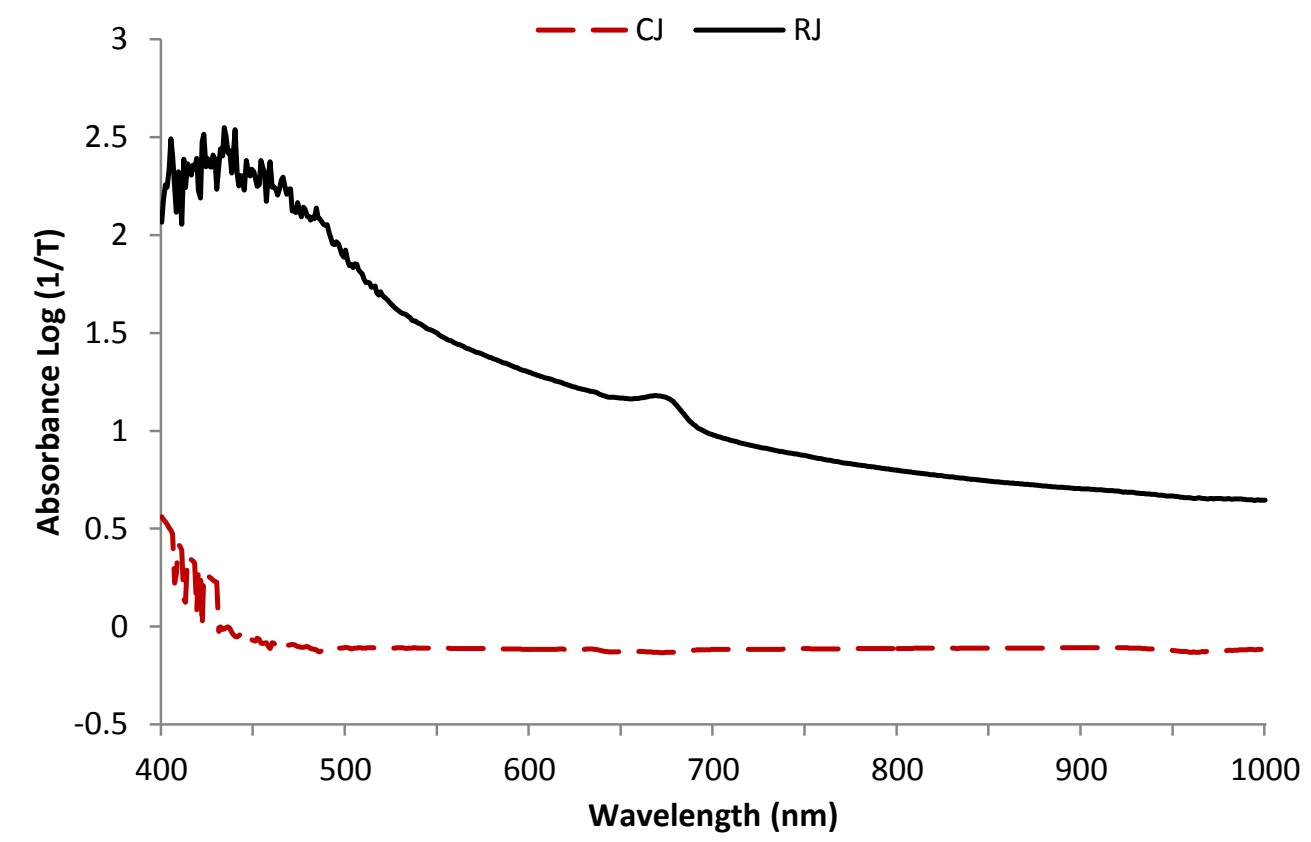

Figure 3.Typical absorbance spectra of raw and clear sugarcane juices $(\mathrm{CJ}=$ clear juice/ $\mathrm{RJ}=$ raw juice)

Table 1. Statistical characteristic of the juice samples

\begin{tabular}{cccccc}
\hline Parameters & Data set & Max & Min & Mean & $\begin{array}{c}\text { Standard } \\
\text { Deviation }\end{array}$ \\
\hline \multirow{2}{*}{ Brix } & Calibration & 25.2 & 19.3 & 22.7 & 1.48 \\
& Prediction & 24.7 & 19.3 & 22.8 & 1.46 \\
\multirow{2}{*}{ Pol } & Calibration & 100.6 & 71.0 & 88.3 & 8.07 \\
& Prediction & 98.8 & 71.7 & 88.8 & 7.31 \\
\hline
\end{tabular}

The statistical characteristics of brix and pol for the juice samples for both calibration and prediction are shown in Table 1. Since, the samples in calibration and validation sets were selected systematically, it was found that the range and mean values 
of brix and pol were almost identical in both data sets. Since the data was collected from different locations throughout the whole harvest season, the brix and pol values in both data sets would be considered sufficient to represent the typical quality values of sugarcane during harvest.

\section{PLS Models Performance}

PLS models were developed to correlate the spectral data with the sugarcane quality components in both calibration and prediction data sets. The performances of the both calibration and prediction models for both juice samples were evaluated using $\mathrm{R}^{2}$, RMSEC and RMSEP (Table 2). Table 2 shows that both calibration and prediction models for clear juice were good with $\mathrm{R}^{2}$ values being 0.84 and 0.85 respectively. For raw juice samples, the performance of calibration models for both brix and pol were good with $\mathrm{R}^{2}$ values being 0.84 for both components. The prediction performance of the clear juice samples was good with $\mathrm{R}^{2}$ values for brix and pol were 0.84 and 0.85 , respectively. For the raw juice samples, the prediction performance was acceptable with $\mathrm{R}^{2}$ values for brix and pol were 0.74 and 0.73 , respectively. The prediction performance of clear juice was better than raw juice because raw juice was an opaque, frothy and viscous liquid owing to the presence of colloidal substance. These substances would absorb energy from light source thus influence the prediction of sucrose content in the juices. In clear juice samples, those colloidal substances were removed through clarification process. However, despite lower accuracy, raw juice samples were still acceptable for field screening as complex sample preparation and processing could be avoided.

Table 2. PLS model performances for both juice samples.

\begin{tabular}{ccccccc}
\hline \multirow{2}{*}{ Samples } & \multirow{2}{*}{ Parameters } & \multirow{2}{*}{ LVs } & \multicolumn{2}{c}{ Calibration } & \multicolumn{2}{c}{ Prediction } \\
\cline { 4 - 7 } & & & $\mathrm{R}^{2}$ & RMSEC & $\mathrm{R}^{2}$ & RMSEP \\
\hline \multirow{2}{*}{ Clear juice } & Brix & 7 & 0.86 & 0.81 & 0.84 & 1.01 \\
& Pol & 8 & 0.88 & 4.01 & 0.85 & 4.49 \\
\hline \multirow{2}{*}{ Raw juice } & Brix & 6 & 0.84 & 0.83 & 0.74 & 0.99 \\
& Pol & 6 & 0.84 & 4.62 & 0.73 & 5.68 \\
\hline
\end{tabular}

It can be seen from Table 2 that pol prediction for clear juice samples was better than brix prediction. This finding is consistent with the study reported by Berding, Brotherton [14]. The performance of prediction models of pol for both juice samples are presented by the scatter plots in Figure 4(a) and (b), respectively. The performances of these models were evaluated by 23 juice samples in the prediction set. The $\mathrm{R}^{2}$ for clear juice and raw juice were 0.87 and 0.75 , respectively. Pol yielded better prediction performance than brix probably because pol was an estimation of sucrose content in juice whereas brix was just an estimation of soluble solids content in the juices. Roggo, Duponchel [15] claimed that spectroscopic method could be an accurate method to determine pol (sucrose content) and brix. The authors also reported that brix and sucrose are highly correlated, where sucrose content in brix was about $80 \%$. This fact explained why pol prediction was better than brix prediction. 

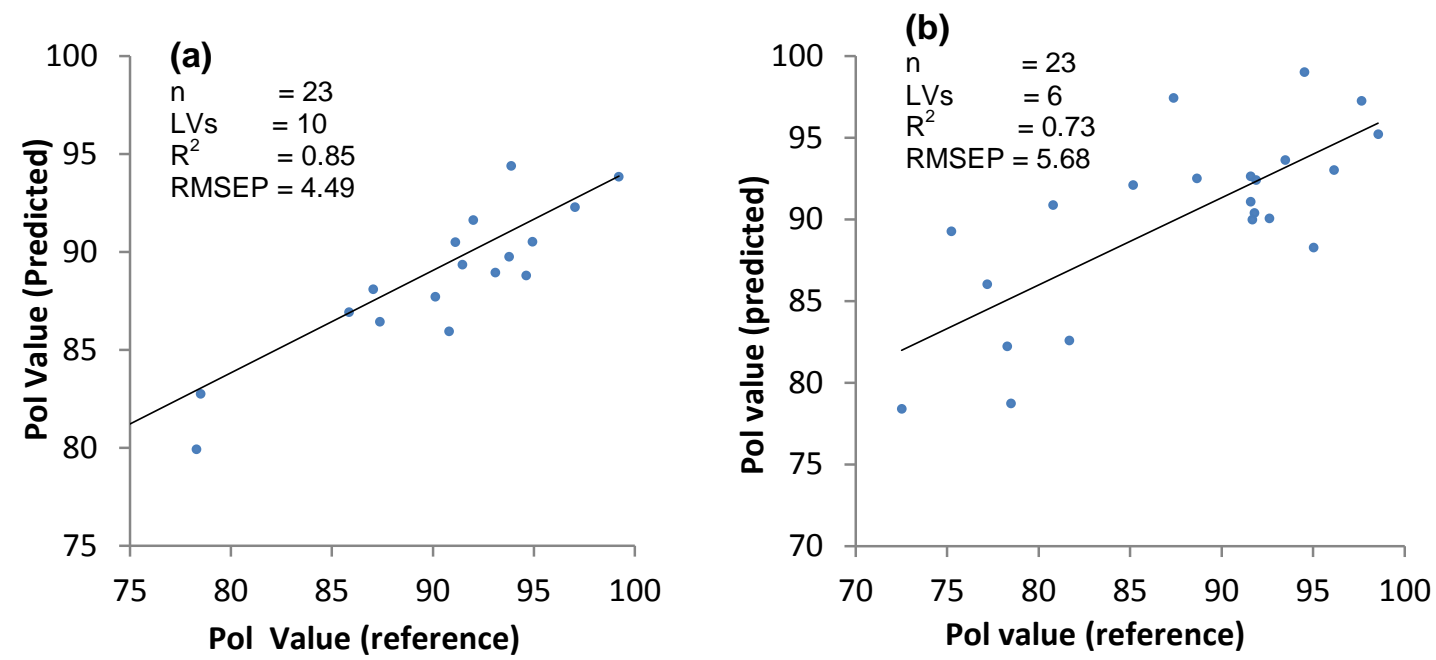

Figure 4. Scatter plots of reference versus predicted pol; (a) clear juice (b) raw juice

\section{CONCLUSIONS}

The results obtained in this study demonstrated the potential of the VNIRS to predict brix and pol content in both clear and raw sugarcane juices. For the clear juice samples, the $\mathrm{R}^{2}$ values for brix and pol prediction were 0.84 and 0.85 , respectively. While for the raw juice samples, the prediction accuracies of brix and pol were slightly lower than the clear juice samples with $\mathrm{R}^{2}$ values of 0.74 and 0.73 , respectively. The results of this study suggest that a low-cost and portable VNIRS could offer the possibility to predict sugarcane quality in the field without the need for costly and laborious analysis using the conventional methods. However, the development of a portable mini sugarcane crusher would be needed to supply juice samples for the instrument in the field. Further studies are also needed in order to improve calibration specificity, accuracy and robustness, and to further interpret and develop new applications of this technique in the sugarcane industry.

\section{ACKNOWLEDGEMENTS}

The authors acknowledge the financial supports provided by Ministry of Higher Education, Malaysia, Universiti Putra Malaysia and National Center for Engineering in Agriculture (NCEA), Toowoomba, Australia. The authors also thank BSES Limited, Bundaberg, for providing samples and equipment.

\section{REFERENCES}

[1] Mehrotra R, Siesler HW. Application of mid infrared/near infrared spectroscopy in sugar industry. Applied Spectroscopy Reviews. 2003;38:307-54.

[2] Valderrama P, Braga JWB, Poppi RJ. Validation of multivariate calibration models in the determination of sugar cane quality parameters by near infrared spectroscopy. Journal of the Brazilian Chemical Society. 2007;18:259-66.

[3] Taira E, Ueno M, Kawamitsu Y. Automated quality evaluation system for net and gross sugarcane samples using near infrared spectroscopy. Journal of Near Infrared Spectroscopy. 2010;18:209-15. 
[4] Walsh KB, Guthrie JA, Burney JW. Application of commercially available, lowcost, miniaturised NIR spectrometers to the assessment of the sugar content of intact fruit. Functional Plant Biology. 2000;27:1175-86.

[5] Nawi NM, Jensen T, Chen G. The application of spectroscopic methods to predict sugarcane quality based on stalk cross-sectional scanning. American Society of Sugar Cane Technologists Journal. 2012;32:16-27.

[6] Mat Nawi N, Chen G, Jensen T. Application of visible and shortwave near infrared spectrometer to predict sugarcane quality from different sample forms. Proceeding of SPIE 8881, Sensing Technologies for Biomaterial, Food, and Agriculture 2013. Yokohama, Japan2013. p. 1-10.

[7] Mat Nawi N, Chen G, Jensen T, Mehdizadeh SA. Prediction and classification of sugar content of sugarcane based on skin scanning using visible and shortwave near infrared. Biosystems Engineering. 2013;115:154-61.

[8] Naes T, Isaksson T, Fearn T, Davies T. A user friendly guide to multivariate calibration and classification. Charlton, Chichester, UK: NIR publications; 2002.

[9] Moghimi A, Aghkhani MH, Sazgarnia A, Sarmad M. Vis/NIR spectroscopy and chemometrics for the prediction of soluble solids content and acidity $(\mathrm{pH})$ of kiwifruit. Biosystems Engineering. 2010;106:295-302.

[10] Wu D, Feng L, Zhang C, He Y. Early detection of Botrytis cinerea on eggplant leaves based on visible and near-infrared spectroscopy. Transactions of the ASABE. 2008;51:1133-9.

[11] Arana I, Jaren C, Arazuri S. Maturity, variety and origin determination in white grapes (Vitis Vinifera L.) using near infrared reflectance technology. Journal of Near Infrared Spectroscopy. 2005;13:349-57.

[12] Cadet F, Offmann B. Direct spectroscopic sucrose determination of raw sugar cane juices. Journal of Agricultural and Food Chemistry. 1997;45:166-71.

[13] Abbott JA, Lu R, Upchurch BL, Stroshine RL. Technologies for nondestructive quality evaluation of fruits and vegetables. Horticultural Reviews: John Wiley \& Sons, Inc.; 2010. p. 1-120.

[14] Berding N, Brotherton GA, le Brocq DG, Skinner JC. Near infrared reflectance spectroscopy for analysis of sugarcane from clonal evaluation trials: II. Expressed Juice. Crop Sciences. 1991;31:1024-8.

[15] Roggo Y, Duponchel L, Huvenne J-P. Quality evaluation of sugar beet (beta vulgaris) by near-infrared spectroscopy. Journal of Agricultural and Food Chemistry. 2004;52:1055-61. 